Bundesgesundheitsbl -

Gesundheitsforsch - Gesundheitsschutz

2002 • 45:364-370 @ Springer-Verlag 2002

Originalien und Übersichtsarbeiten

C. Meyer ${ }^{1}$ - G. Rasch ${ }^{1}$ - B. Keller-Stanislawski ${ }^{2}$ N. Schnitzler ${ }^{3}$

${ }^{1}$ Robert Koch-Institut, Berlin

2 Paul-Ehrlich-Institut, Langen

${ }^{3}$ Bundesministerium für Gesundheit, Bonn

\title{
Anerkannte Impfschäden in der Bundesrepublik Deutschland 1990-1999
}

Beitrag zur Transparenz und Vereinheitlichung bei der Bewertung von Impfkomplikationen leisten.

\section{Schlüsselwörter}

Impfkomplikationen . Entschädigung bei Impfkomplikationen . Surveillance von Impfkomplikationen rorgungsverwaltungen der Länder zeigt dass Entschädigungen und Anträge auf Entschädigungen zu Impfkomplikationen für den Zeitraum von 1991 bis 1999 weiterhin rückläufig sind. Im Zeitraum von 1976 bis 1990 wurden 1139 von 4569 gestellten Anträgen anerkannt, im Zeitraum von 1991 bis 1999 hingegen nur 389 von 2543 Anträgen. 38\% aller Entschädigungsverfahren beziehen sich auf die seit 1982 nicht mehr durchgeführte Pockenimpfung. Regionale Unterschiede legen die Vermutung nahe, dass verfahrensbedingte Eigenheiten des Gesundheitswesens und gesamtgesellschaftliche Veränderungen Grundlage von unterschiedlichen Häufigkeiten und Raten sind. Epidemiologische oder kausale Fragestellungen sind aus den Daten der Versorgungsverwaltungen zu Entschädigungen nach Impfkomplikationen nicht zu beantworten. Eine valide aussagekräftige Analyse von Impfkomplikationen erfordert eine postvakzinale Untersuchung des Einzelfalles. Hier sind in Zukunft aus den Meldedaten des Infektionsschutzgesetzes und anderen neu zu implementierenden Datengrundlagen (z. B.DLDB (Data Linked Data Bases) bei Krankenkassen) Erkenntnisse zu erwarten. Eine zentrale Gutachterkommission zu Impfkomplikationen könnte einen wertvollen mpfungen gehören zu den kosteneffektivsten und sichersten Maßnahmen zur Verhinderung von Infektionskrankheiten im Gesundheitswesen. Der Nutzen von Schutzimpfungen wiegt im Rahmen der Entscheidung für eine Impfung die seltenen Risiken und Kosten des Impfstoffs sowohl für den einzelnen Geimpften als auch für die Bevölkerung auf. Impfungen haben beim Rückgang von impfpräventablen Erkrankungen in Deutschland einen wertvollen Beitrag geleistet und sind auch in Zukunft die vordringlichste Maßnahme der primären Prävention dieser Erkrankungen.

Prinzipiell kann jede Impfung zu Komplikationen führen, wobei für unterschiedliche Impfungen spezifische Komplikationen $\mathrm{zu}$ beschreiben sind. Impfempfehlungen werden nur für Impfstoffe ausgesprochen, bei denen Komplikationen durch die Erkrankung deutlich häufiger sind als die sehr selten auftretenden Impfkomplikationen, so dass eine Risiko-Nutzen-Abwägung im- mer zugunsten des Impfstoffs ausfällt. Doch kein Impfstoff kann trotz intensiver Überwachung und Qualitätssicherung in Herstellung, Zulassung und Anwendung vollständig nebenwirkungsfrei sein. Als „normale“ Reaktion des Organismus auf den Impfstoff werden lokale und allgemeine Symptome beobachtet. Hiervon zu unterscheiden sind die seltenen, durch den Impfstoff hervorgerufenen gesundheitlichen Störungen im Sinne von Komplikationen, die in einem ursächlichen Zusammenhang mit der Impfung stehen.

\section{„Vermutete Impfkomplikationen können zu dramatischen Ein- brüchen in der Impfakzeptanz führen."}

Da Impfungen neben dem individuellen Gesundheitsschutz auch im Interesse der gesamten Bevölkerung stehen, werden auftretende Komplikationen im Rahmen von staatlichen Versorgungsleistungen entschädigt, wobei zur Anerkennung eines Impfschadens in Deutschland der mögliche Zusammenhang mit der Impfung ausreicht $(\$ \$ 60$, 61 Infektionsschutzgesetz, IfSG). Mit dem erfreulichen Rückgang einiger In-

Dr. Christiane Meyer

Robert Koch-Institut, Nordufer 20,13353 Berlin, E-Mail: MeyerC@rki.de 


\section{Meyer · G. Rasch · B. Keller-Stanislawski N.Schnitzler}

\section{Vaccine-Related Injuries in Germany from 1990 to 1999}

\section{Abstract}

The decrease in infectious diseases preventable by immunisation and the absence of complications caused by these diseases leads to an increased awareness of vaccineassociated adverse events. The analysis of a survey of the vaccine injury compensation data from the German Bundesländer shows the decrease in accepted and demanded compensation from 1991 to 1999. From 1976 to 19901139 of 4569 demands were accepted, whereas from 1991 to 1999, acceptance of only 389 of 2543 demands was reported. In all, $38 \%$ of the accepted compensations refer to the smallpox vaccine which is not longer recommended by the STIKO (Permanent Vaccination Commission in Germany) since immunisation against smallpox was stopped in the 1980s. Regional differences show that process elements of the German healthcare system as well as political and social reasons express most of the differences in rates and prevalence of vaccine associated adverse events. Epidemiological questions and questions of causality cannot be answered by the analysis of data collected in vaccine injury programs. Valid analysis needs a register of individual documented cases of vaccine adverse events. The surveillance of adverse events following immunisation will make progress by the analysis of data reported according to the Protection against Infection Act (IfSG) and by further surveillance systems that should be implemented in the near future. A centralised commission with expert opinion concerning causality could increase transparency and homogeneity within judgement and documentation of vaccine associated adverse events.

\section{Keywords}

Vaccine-Associated Adverse Events .

Vaccine Injury Compensation .

Surveillance for Vaccine-Associated Adverse Events fektionskrankheiten und deren Komplikationen drängen Impfkomplikationen und Nebenwirkungen in den Vordergrund einer öffentlichen Diskussion und beeinflussen das Impfverhalten im Sinne einer sinkenden Impfakzeptanz (vgl. Abb. 1) [1]. Wegen der sinkenden Inzidenz impfpräventabler Erkrankungen richtet sich das Augenmerk der öffentlichen Diskussion zunehmend auf potenzielle oder auch vermutete Impfkomplikationen und kann, wie Erfahrungen mit dem Pertussisimpfstoff und erst jüngst mit dem Masernimpfstoff in England zeigen, zu dramatischen Einbrüchen in der Akzeptanz von Impfungen führen.

Da Impfungen in der Regel vollständig gesunden Kindern und Erwachsenen verabreicht werden, deren individueller Gesundheitsschutz von großem Interesse ist, gilt es, selten auftretende unerwünschte Ereignisse besonders $\mathrm{zu}$ beobachten, zu dokumentieren, zu bewerten und im Schadensfall gegebenenfalls auch zu entschädigen.

Die Sicherheit eines Impfstoffes wird heute in standardisierten Verfahren der europäischen und nationalen Zulassungsbehörden bereits vor der $\mathrm{Zu}$ lassung genauestens geprüft (CPMP: Committee for Proprietary Medicinal Products, EMEA: European Medicines Evaluation Agency). Sehr selten auftretende Impfkomplikationen $(\leq 1: 10.000$ Impfungen) sind in solchen klinischen Zulassungsstudien nicht immer vollständig auszuschließen [2, 3], weshalb die Überwachung unerwünschter $\mathrm{Ne}$ benwirkungen von Impfstoffen in Deutschland nach ihrer Zulassung - bisher als Spontanerfassung durch die Ärzte (verankert in den Berufsordnungen der Ärzte und über das Arzneimittelrecht [AMG]) und die überwachenden Behörden (Paul-Ehrlich-Institut) durchgeführt - von besonderer Bedeutung ist. National und international gibt es außerdem weitere epidemiologische Überwachungssysteme zur Erfassung und Bewertung von Impfkomplikationen. Hier sind passive und aktive Surveillancesysteme (Vaccine Advers Event Reporting System-USA, Großbritannien, Kanada) $[4,5,6]$ von epidemiologischen und klinischen Studien [7] sowie von so genannten verknüpften Datenbanken (Data Linked Data Bases) zu unterscheiden $[8,9]$. Passive Surveillancesysteme zeigen die bekannten Probleme von Un- vollständigkeit, Underreporting und beschränkter Validität der Daten sowie Verzerrungen durch unterschiedliches Meldeverhalten und unterschiedliche Wahrnehmung (recall bias). Es konnte jedoch gezeigt werden, dass der Nutzen passiver Surveillancesysteme im Rahmen der Überwachung und der Hypothesengenerierung zu Impfkomplikationen unbestritten ist. Ein aktuelles Beispiel für die Effektivität dieser Überwachung sind die Rücknahme eines FSME (Frühsommer-Meningoenzephalitis)Impfstoffs oder die Erkenntnisse zu Invagination (Einstülpung eines Darmabschnittes in den folgenden Darmabschnitt) nach oraler Rotavirusimpfung $[10,11,12,13]$.

Da Impfungen zusätzlich zu ihrem individuellen Nutzen für den Geimpften auch von bevölkerungsmedizinischem Interesse sind (Herdimmunität), haben eine Reihe von Ländern seit Beginn der 196oer-Jahre Programme zur Entschädigung von Impfkomplikationen verabschiedet [14]. In der DDR bestanden seit 1949 Rechtsverordnungen zur Entschädigung bei Impfkomplikationen, die 1964/65 im Gesetz über den Verkehr mit Arzneimitteln und im Gesetz zur Verhütung und Bekämpfung übertragbarer Krankheiten gesetzlich geregelt und 1974/75 durch weitere Durchführungsbestimmungen erweitert wurden $[15,16$, 17]. In der Bundesrepublik Deutschland wurde ein solcher Entschädigungsanspruch in einem Urteil des Bundesgerichtshofes erstmalig 1953 bestätigt. Seit 1961 besteht in der Bundesrepublik eine im Bundes-Seuchengesetz und seit 1. Januar 2001 im IfSG erneut gesetzlich verankerte Entschädigungsmöglichkeit bei Auftreten von Impfkomplikationen (\$\$60-65 IfSG). Zwölf weitere Länder verfügen über ähnliche Programme zur Entschädigung: Dänemark (1972), England (1979), Frankreich (1964), Italien (1972), Norwegen (1995), Schweden (1978), Schweiz (1970), Kanada (1985), USA (1988), Neuseeland (1974), Japan (1970) und Taiwan (1988) [14]. Die Ausgestaltungen der Programme sind hinsichtlich der Entschädigungsleistungen, der Entschädigungsgründe, der Finanzierung, des Begutachtungsprozesses, der Auswahlkriterien und weiterer Prozessmerkmale unterschiedlich, so dass Daten aus solchen Programmen zu Entschädigungen von Impfkomplikationen schwer zu vergleichen sind. 


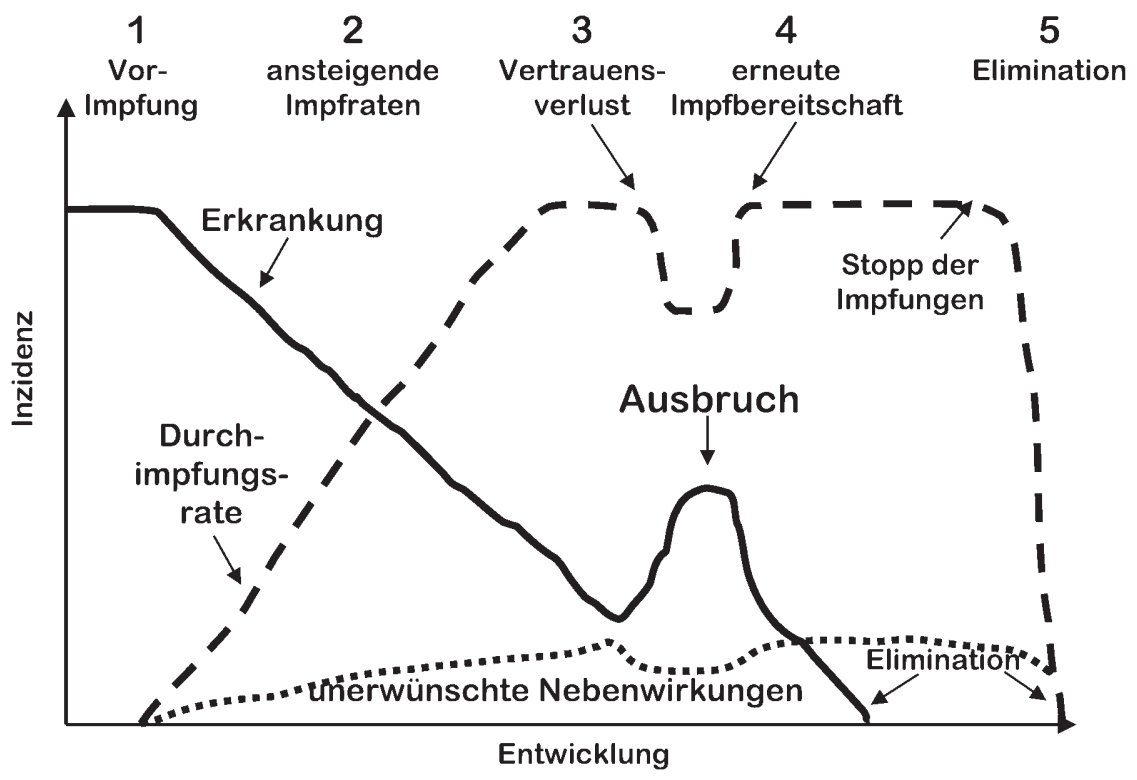

Abb. $1 \Delta$ Unterschiedliche Phasen der Wahrnehmung von Erkrankungs- und Impfkomplikationen im Verlauf der Elimination einer impfpräventablen Erkrankung [1]

Entschädigungsleistungen im Rahmen solcher Programme ermöglichen, da sie aufgrund der staatlichen Verantwortung für den Schutz des Individuums erfolgen, Leistungen bereits bei einem für möglich erachteten Zusammenhang von Impfung und Komplikation. Daten, die auf der Grundlage der Entschädigungen nach Impfkomplikationen erhoben werden, sind für die Bewertung der Kausalitäten von Impfkomplikationen sowie zur epidemiologischen Nutzung für die Berechnung von Inzidenzen und Prävalenzen deshalb nur begrenzt nutzbar und müssen besonders vorsichtig interpretiert werden $[18,19]$. Wie andere passive Surveillancesysteme auch können sie allenfalls als Frühwarnsysteme sowie zur Hypothesengenerierung dienen und passive Surveillancesysteme ergänzen.

\section{„Daten über Impfschäden, die aufgrund von Entschädigungs- leistungen erhoben werden, sind nur begrenzt nutzbar."}

Ohne effektive, valide und vollständige Erfassungssysteme zur Aufdeckung von Impfkomplikationen droht die Diskussion über Nebenwirkungen von Impfungen zu Verwirrung und zur Ablehnung von Impfungen zu führen (vgl. Abb. 1). Eine wissenschaftliche Analyse der Komplikationen ist auf Dauer nur über die aktive Einzelfallanalyse aller postvakzinalen Komplikationen möglich.

\section{Daten zu Impfkomplikationen in Deutschland}

Von 1971 bis 1990 wurden in der Bundesrepublik dem Bundesgesundheitsamt (BGA) jährlich die Daten der anerkannten Impfkomplikationen über die Versorgungsverwaltungen der Länder gemeldet und in einem Impfschadensregister zusammengeführt $[20,21]$. Als Bezugsgrößen lagen die Daten der durch den öffentlichen Gesundheitsdienst durchgeführten Impfungen vor. Eine Berechnung der Inzidenz von Impfkomplikationen war aufgrund der Unvollständigkeit von Nenner und Zähler nicht möglich. Seit 1965 wurde in der DDR die Mehrzahl aller atypischen Impfverläufe erfasst [15]. Seit dem Jahr 1988 verpflichten die Berufsordnungen im Bundesgebiet alle Ärzte zur Meldung unerwünschter Arzneimittelnebenwirkungen an die Arzneimittelkommission der deutschen Ärzteschaft. Die Meldungen, die Impfstoffe betreffen, werden in anonymisierter Form an das Paul-EhrlichInstitut (PEI) weitergeleitet. Spontanmeldungen von Angehörigen der Gesundheitsberufe und von Impfstoffherstellern werden hier ebenfalls zusammengeführt. Impfstoffhersteller sind aufgrund des Arzneimittelgesetzes und in Übereinstimmung mit der EU-Richt- linie 75/319/EWG verpflichtet, alle weltweit aufgetretenen schwerwiegenden unerwünschten Arzneimittelnebenwirkungen (UAW) und Verdachtsfälle den zuständigen Behörden zu melden. Am PEI liegt somit eine vollständige Datenbank zum Nebenwirkungsspektrum der Impfstoffe vor, die eine Überwachung ermöglicht und geeignet ist, schwerwiegende Nebenwirkungen im Zusammenhang mit Impfungen aufzuzeigen, Hypothesen über die Ursachen von Impfkomplikationen zu generieren oder einen vermuteten Zusammenhang auszuschließen [22].

Wie bereits erwähnt, besteht in der Bundesrepublik seit 1961 zunächst im Bundes-Seuchengesetz und seit dem 1. Januar 2001 im IfSG ein gesetzlich verankerter staatlicher Entschädigungsanspruch nach Feststellung eines Impfschadens (\$\$60-65 IfSG). Im IfSG ist auBerdem die Meldung des Arztes an das zuständige Gesundheitsamt bei Verdacht auf Vorliegen einer über das übliche Maß hinausgehenden Impfreaktion geregelt (\$11 Abs. 2). Hintergrund dieser Meldepflicht ist die Schaffung der Möglichkeit einer Beratung durch das Gesundheitsamt im Rahmen der differenzialdiagnostischen Maßnahmen und Bewertungen sowie die Schaffung einer fundierten Datenbasis zu Impfkomplikationen. Die Meldedaten werden anonymisiert dem PEI zugesandt. Die Meldung umfasst (\$11 Abs. 2) die Bezeichnung des Produkts, den Namen des pharmazeutischen Unternehmens, Chargenbezeichnung, Zeitpunkt der Impfung, Beginn der Erkrankung, Geburtsdatum des vermeintlich Geschädigten, Geschlecht, den ersten Buchstabe des Nachnamens und ersten Buchstabe des Vornamens (vgl. Meldebogen unter http://www.pei.de oder http://www.rki.de).

\section{Methode der Datenerhebung}

Im Jahr 2000 wurde vom Bundesministerium für Arbeit und Sozialordnung in Zusammenarbeit mit dem Bundesministerium für Gesundheit (BMG), dem Robert Koch-Institut (RKI) und dem PEI eine Befragung der Versorgungsverwaltungen der Länder veranlasst. Diese wurden hinsichtlich der Häufigkeit und Art der nach Impfungen auftretenden Gesundheitsstörungen und den erfolgten Impfentschädigungen im Zeitraum von 1991 bis 1999 befragt. Mittels eines 


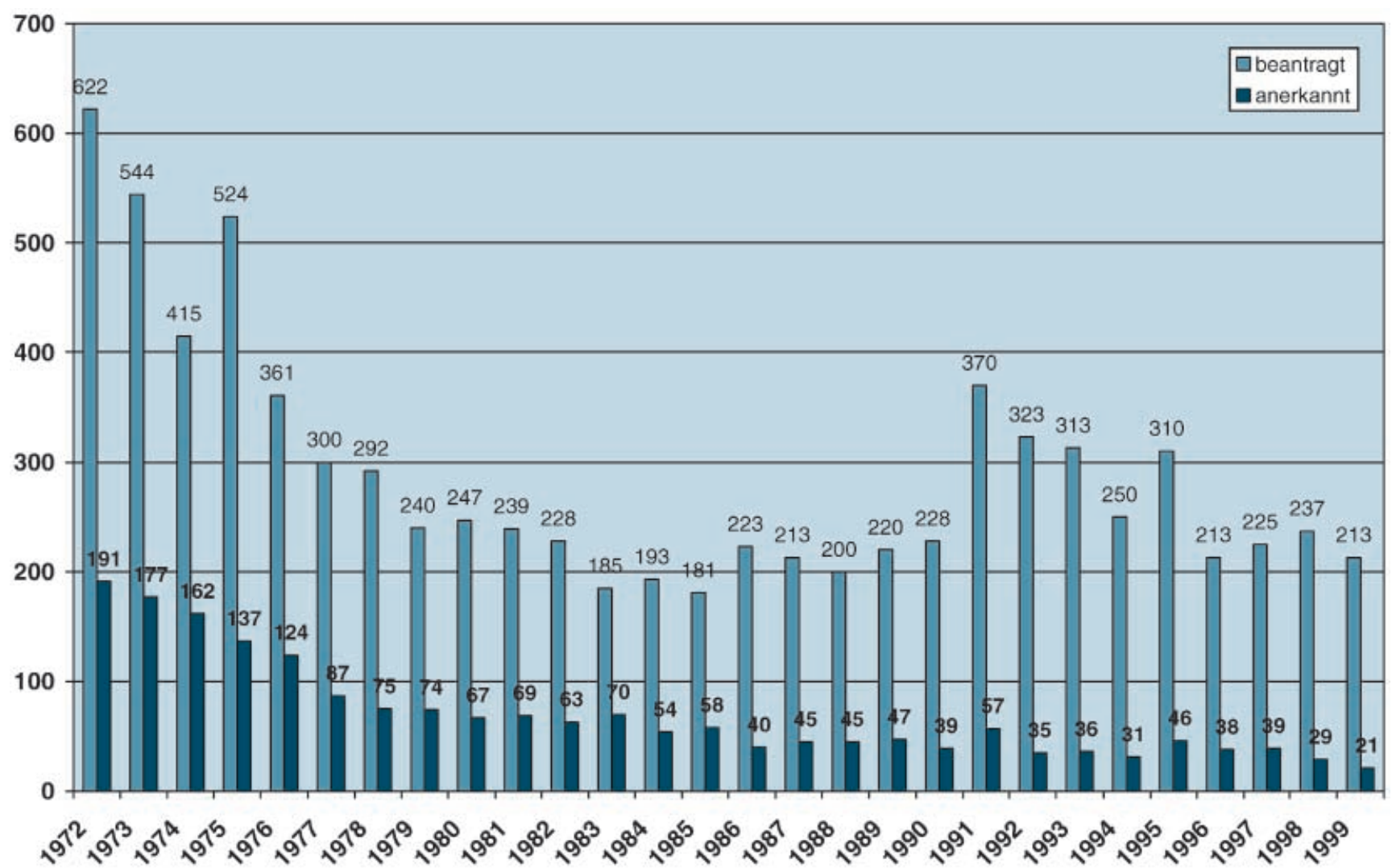

Abb.2 $\triangle$ Anerkennung von Impfschäden in Deutschland 1972-1999, 1972-1990 BRD;1991 -1999 alte und neue Bundesländer

nicht standardisierten Fragebogens wurden aggregierte Daten für den interessierenden Zeitraum erhoben und die Daten nach Abschluss der Erhebungsphase dem RKI 2000 zur Auswertung zugesandt. Es zeigte sich, dass die auf diese Weise erhobenen Daten (ausschließlich aggregierte, nicht standardisierte Daten) zwar eine Beschreibung der Situation ermöglichen, jedoch keine differenzierten Analysen bezüglich epidemiologischer und kausaler Fragestellungen erlauben. Im Folgenden werden deshalb die Impfschadensmeldungen nur im Hinblick auf ihre Häufigkeit, den zeitlichen Verlauf und die regionalen Unterschiede dargestellt.

\section{Ergebnisse der Datenerhebung}

Der Auswertung liegen Daten aus 16 Bundesländern zu Grunde, die jedoch bezüglich Dokumentation, Zeitraum, Bewertung der Gesundheitsstörung, verwendeter Definitionen sowie Differenzierungsgrad zum Teil sehr unterschiedlich sind.

Die Abb. 2 zeigt den zeitlichen Verlauf für gestellte Anträge und anerkann- te Impfschäden seit 1972 in den alten Bundesländern aufgrund einer Impfschadensmeldung. Nach der Wiedervereinigung stehen seit 1991 gemeinsame Daten für die alten und neuen Bundesländer zur Verfügung. Eindrücklich lässt sich der stetig abnehmende Trend bis zu Beginn der 1990er-Jahre zeigen. Dieser setzt sich auch im Verlauf der 1990erJahre fort. Aufgrund des Bevölkerungswachstums durch die Wiedervereinigung ist in diesen Jahren ein Anstieg der Antragstellungen zu beobachten. Die Zahl der Anerkennungen von Impfkomplikationen ist jedoch auch nach 1991 weiter rückläufig.

\section{„Die Anzahl der Anträge auf Anerkennung von Impfschäden ist seit Anfang der 1990er-Jahre rückläufig."}

Abbildung 3 zeigt diesen rückläufigen Trend für die Jahre 1991 bis 1999 in Bezug auf 100.000 Einwohner (Datengrundlage: 13 Bundesländer). Eine Standardisierung auf durchgeführte Impfungen oder geimpfte Personen ist wegen fehlender Daten nicht möglich, so dass ein Bevölkerungsbezug die einzige Möglichkeit darstellt, Verzerrungen aufgrund von unterschiedlichen Bezugsgrößen zu bereinigen. Wurden 1991 noch 0,081 Impfkomplikationen für 100.000 Einwohner anerkannt, so ist diese Rate 1999 auf 0,029/100.000 gefallen. In der Bundesrepublik werden pro Jahr im Berichtszeitraum im Mittel o,053 Impfkomplikationen/100.000 Einwohner entschädigt, wobei hier zwischen den neuen und alten Bundesländern ein Unterschied zu beobachten ist (alte Bundesländer 0,046/100.000; neue Bundesländer 0,099/100.000). In den Jahren 1991 bis 1999 wurden in Deutschland 2543 Anträge auf Anerkennung eines Impfschadens gestellt (Datengrundlage: 16 Bundesländer). Von diesen wurden 389 Anträge anerkannt (vgl. Abb. 4). Dies entspricht einer Anerkennungsrate von insgesamt $15,2 \%$ (alte Bundesländer 15,3\%; neue Bundesländer 15,2\%). $32,8 \%$ (833) der gestellten Anträge wurden in den neuen Bundesländern bearbeitet.

Im Rahmen der Frage von Impfkomplikationen interessiert, welche Gesundheitsstörungen für welche Impf- 


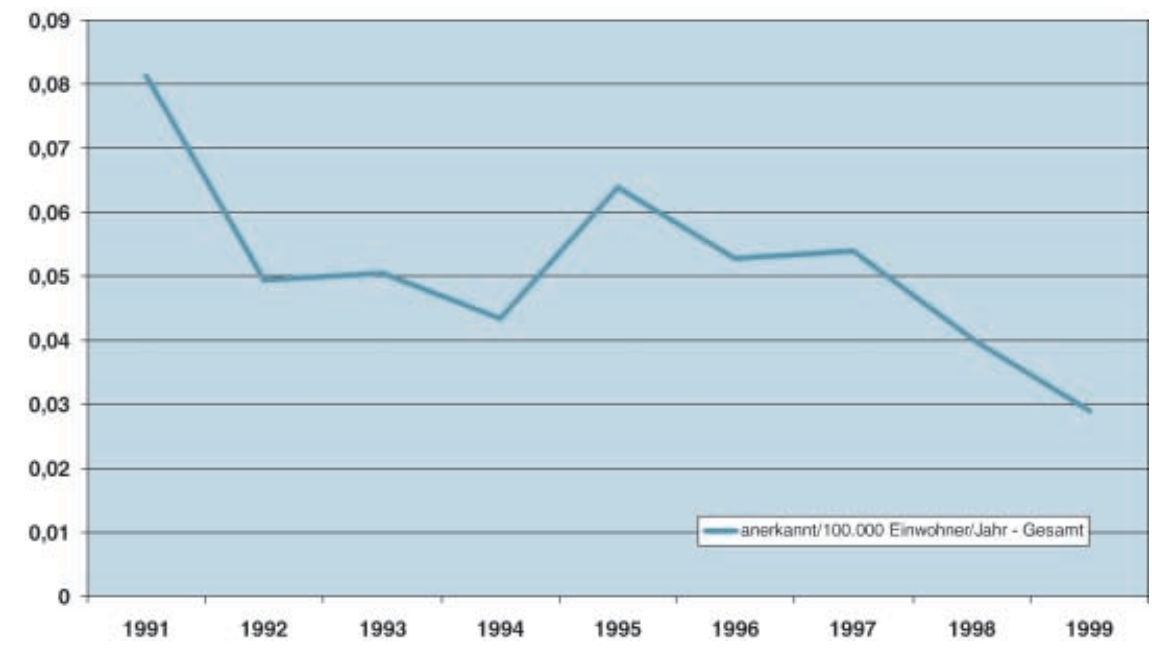

Abb. $3 \Delta$ Anerkannte Impfkomplikationen in Deutschland pro 100.000 Einwohner/Jahr

stoffe auftreten und entschädigt werden. Hierzu liegen für den Zeitraum von 1991 bis 1999 nur aus sechs Bundesländern differenzierte Angaben vor. Werden alle berichteten Impfkomplikationen über der Zeitraum 1972 bis 1999 zusammengefasst, zeigt sich die in Abb. 5 dargestellte Verteilung. Die Verteilung der Entschädigungen nach Impfstoffarten (Abb. 5) zeigt, dass in 64,7\% der Fälle eine Gesundheitsstörung nach Pockenschutzimpfung, in 6,6\% der Fälle nach Impfung mit BCG (Bacille-CalmetteGuerin-Vakzine, Tuberkulose-Vakzine) und in $8,1 \%$ der Fälle eine Gesundheitsstörung nach einer Impfung gegen Poliomyelitis (OPV) entschädigt wird. Diese Impfungen sind von der STIKO seit längerem nicht mehr empfohlen, die Pockenschutzimpfung wird seit 1982 in Deutschland nicht mehr durchgeführt. Auf diese Impfungen entfällt jedoch der größte Teil der anerkannten Impfschäden. Erwartungsgemäß betrifft ein weiterer großer Anteil der Entschädigungen Gesundheitsstörungen infolge einer Impfung mit dem Ganzkeim-PertussisImpfstoff, der ebenfalls von der STIKO nicht mehr empfohlen wird. Gesundheitsstörungen in Folge der Tetanusimpfung sind ebenfalls häufiger gemeldet worden. Die Betrachtung der Daten aus einem aktuelleren Zeitraum (1991 bis 1999, sechs Bundesländer (BadenWürttemberg, Bayern, Berlin, Brandenburg, Nordrhein-Westfalen, Thüringen) $\mathrm{n}=228$ ) zeigt jedoch deutlich, dass dieses Muster auch in den 1990er-Jahren bestehen blieb (35\% Pocken, 13\% BCG, 12\% Polio, 9\% Tetanus, 10\% DPT (Diph-
therie-Pertussis-Tetanus) bzw. DPTPolio, 4\% FSME, 3\% MMR (Masern, Mumps, Röteln)/Masern, $2 \%$ Keuchhusten, $2 \%$ Hepatitis, 3\% Vierfachkombination, $1 \%$ Fünffachkombination, $6 \%$ sonstige), obwohl die Pockenimpfung nicht mehr und die BCG-Impfung nur noch selten durchgeführt wurden.

In der Öffentlichkeit werden insbesondere Komplikationen nach Verwendung von Masern-Mumps-Rötelnimpfstoff (MMR-Impfstoff) diskutiert. Nur 3\% (sieben Fälle) der von sechs Bundesländern gemeldeten Komplikationen betreffen diese Impfung. Ausgehend von einer vorsichtigen Schätzung und bei einer angenommen Durchimpfungsrate für die erste Masern-Mumps-Rötelnimpfung von circa 75 bis $80 \%$, sind in diesen Bundesländern 1,7 bis 1,9 Millionen Dosen Impfstoff im Jahr verimpft worden [23]. Dies entspräche einer maximalen Rate von sieben Komplikationen/16 Millionen Impfstoffdosen. Die berichteten Gesundheitsstörungen dieser sieben Kinder betreffen: eine vollständig ausgeheilte Meningitis, einmal Impfmasern, einen unklaren fortschreitenden Hirnabbauprozess, eine spastische Diplegie und eine Halbseitenlähmung mit Gangstörung (zwei ohne nähere Angaben). Eine nachträgliche kausale Bewertung dieser Ereignisse ist nicht möglich. Systematische Analysen oder Kausalitätsbewertungen anhand der von der WHO definierten Kausalitätskriterien [24] sowie epidemiologische Analysen erlaubt das vorhandene Datenmaterial ebenfalls nicht.

\section{Bewertung und Ausblick}

Die vorliegende Arbeit hat aus einer Befragung des Bundesministeriums für Arbeit und Sozialordnung in Zusammenarbeit mit dem BMG eine darstellende Bewertung der in Deutschland von 1991 bis 1999 anerkannten Entschädigungen wegen Impfkomplikationen vorgenommen.

Gegenüber den Auswertungen des Impfschadensregisters des BGA [20, 21] zeigt sich in den vorliegenden Daten eine weitere Abnahme sowohl der Anzahl der gestellten Anträge als auch der Zahl der anerkannten Impfschäden (von 1976 bis 1990: 1139 Anerkennungen bei 4569 Anträgen; von 1991 bis 1999: 389 Aner-

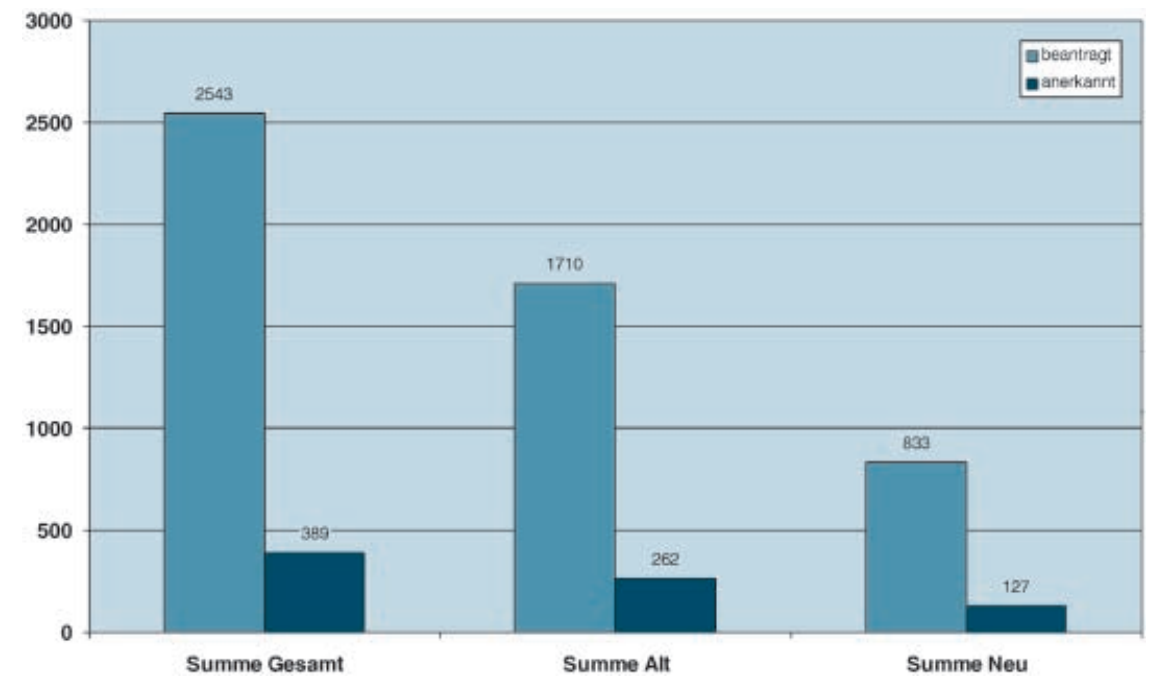

Abb. 4 Anzahl der beantragten und anerkannten Impfschäden 1991-1999 (Alt alte Bundesländer, Neu neue Bundesländer) 


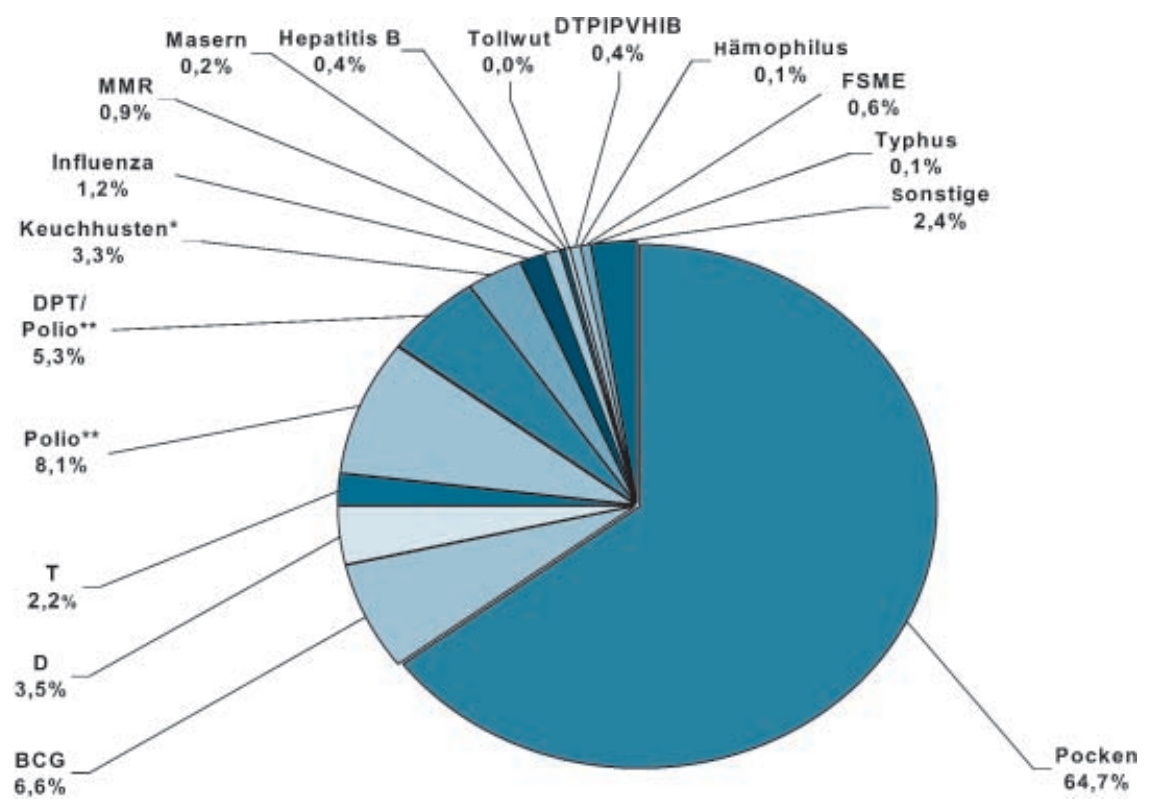

Abb.5 Verteilungen der anerkannten Impfkomplikationen nach Art des Impfstoffes 1972-1999.

*aP und Ganzkeim. **OPV und IPV (OPV orale Poliomyelitis-Vakzine, IPV inaktivierte

Poliomyelitis-Vakzine, aP Pertussis-Antigen, BCG Tuberkulose, D Diphtherie, $T$ Tetanus, DPTpolio Diphtherie-Pertussis-Tetanus-Polio, MMR Masern-Mumps-Röteln, HIB Hämophilus, FSME Frühsommer-Meningoenzephalitis)

kennungen bei 2543 Anträgen). Wurden in den Vorjahren noch 24,9\% der Anträge auf Entschädigung nach Impfkomplikationen anerkannt, so waren es von 1991 bis 1999 nur noch ca. 15,2\%. Beim Vergleich der Daten aus diesen Zeiträumen sind jedoch eine Reihe von methodischen Schwierigkeiten zu berücksichtigen. Aktuell liegen veränderte Impfstoffe vor, Impfempfehlungen sind gegebenen epidemiologischen Situationen und neuen Impfstoffentwicklungen angepasst worden, Begutachtungspraxis und Rechtsprechung sind einem Wandel unterworfen, Impfdosen und Zeitpunkte sind verändert, Inanspruchnahme und Versorgung im Gesundheitswesen haben sich gewandelt und Bevölkerungszahlen sowie Durchimpfungsraten sind aufgrund demographischer, politischer und anderer Gründe erheblich modifiziert. Ohne eine stabile Bezugsgröße (Zähler: geimpfte Personen, verimpfte Dosen) und eine Differenzierung nach Impfstoffen kann eine valide Bewertung der vorliegenden Daten nicht vorgenommen werden.

Zur Abschätzung der Häufigkeit von Impfkomplikationen sind deshalb die gemeldeten Fälle auf die verimpften Dosen oder aber auf die geimpften Personen zu beziehen. Da diese Daten nicht vorliegen, wird in der vorliegenden Auswertung als angleichende Bezugsgröße die Bevölkerung eines Bundeslandes am Ende eines Berichtsjahres gewählt, um eine Verzerrung über unterschiedliche Zähler zu vermeiden. Unterschiedliche Durchimpfungsraten in den Bundesländern gehen in eine solche Betrachtung jedoch nicht ein. Aus dem Vergleich der Daten der neuen und der alten Bundesländer ergeben sich Hinweise darauf, dass die aggregierten Daten aus den Versorgungsverwaltungen eher verfahrensbedingte Eigenheiten des Gesundheitswesens (Prozesselemente) und des gesellschaftlichen Umfeldes darstellen, als dass sie für epidemiologische oder kausale Fragestellungen im Rahmen von Impfkomplikationen $\mathrm{zu}$ verwenden sind. Im Rahmen der Plausibilitätsprüfung der Daten zeigten sich erhebliche Differenzen zwischen den alten und neuen Bundesländern. Diese Unterschiede konnten durch Nachbefragungen gemindert werden und lassen sich z. B. durch unterschiedliche Vorgehensweisen bei der Datenerhebung erklären. Unterschiedliche Inanspruchnahme von staatlichen Versorgungsleistungen sowie unterschiedliches Antragsverhalten können ebenfalls eine Rolle spielen, zumal nach der Wiedervereinigung der beiden deutschen Staaten eine Neubeurteilung der Impfkomplikationsfälle nach Bundes-Seuchengesetz erfolgte. Gleichzeitig erlebten die neuen Bundesländer nach 1990 eine Welle von Anträgen zu vermuteten Impfkomplikationen, deren Impfereignis schon Jahre zurücklag. Soweit möglich, wurden diese möglichen systematischen Verzerrungen in der Datenauswertung berücksichtigt. Da diese jedoch nicht von vornherein Gegenstand der Befragung waren, ist eine vollständige Kompensation dieser Effekte aufgrund der vorliegenden Daten nicht möglich.

Es konnte auch gezeigt werden, dass noch in den 199oer-Jahren die Hälfte der Entschädigungsleistungen auf Impfungen entfielen, die von der STIKO seit längerem nicht mehr empfohlen und daher nicht mehr durchgeführt wurden. Besonders ins Auge fallen hier Entschädigungen im Zusammenhang mit der seit 1982 nicht mehr empfohlenen Pockenschutzimpfung (38\% der Entschädigungen von 1991 bis 1999). Hier spielen wahrscheinlich Migrationsprozesse eine nicht unerhebliche Rolle. Entschädigungsleistungen nach Pockenschutzimpfungen wurden möglicherweise unter Berücksichtigung der damals realisierten Pflichtimpfung auch in der Bundesrepublik Deutschland großzügiger gehandhabt. So stellen die beschriebenen Daten eher die vorhandene Begutachtungspraxis dar als die wirkliche Auftretenshäufigkeit von Impfkomplikationen. Da ein großer Teil der für Entschädigungsleistungen verantwortlichen Impfungen heute von der STIKO nicht mehr empfohlen und in der täglichen Impfpraxis nicht mehr angewandt wird (BCG, OPV, Ganzkeim-Pertussisimpfstoff), ist in Zukunft mit einem weiteren Rückgang der Entschädigungsleistungen aufgrund von Impfkomplikationen zu rechnen.

Eine valide, aussagekräftige Analyse von Impfstoffkomplikationen ist ausschließlich über Einzelfallanalysen in entsprechenden Registern und Erhebungen möglich. Es sollten unbedingt Daten zu Alter und Geschlecht der Person, zum verabreichten Impfstoff, gleichzeitig verabreichten anderen Impfstoffen und bestehenden Begleiterkrankungen, zu weiteren anamnestischen Daten und Risiken, zu Impfzeitpunkt, Impfdosis, Impfanamnese, Art, Ausmaß und Verlauf der Gesundheits- 
störung und zur Kausalität bestehen. Zusätzlich sind epidemiologische Daten zum Zähler (z. B. Anzahl der verimpften Dosen, Anzahl der geimpften Personen) unabdingbar, um Aussagen über die Inzidenz und Prävalenz von Impfkomplikationen treffen und diese national und international vergleichen und bewerten zu können. Daten aus Programmen zur Entschädigung von Impfkomplikationen können dazu dienen, die Plausibilität anderer bestehender passiver Surveillancesysteme $\mathrm{zu}$ überprüfen und diese gegebenenfalls nach Durchführung einer Capture-Recapture-Analyse zu ergänzen [19].

Das neue Infektionsschutzgesetz mit der Meldepflicht einer über das übliche Ausmaß hinausgehenden Impfreaktion setzt hier neue Maßstäbe. Besonders hervorzuheben ist in diesem $\mathrm{Zu}$ sammenhang, dass durch die Meldung an das zuständige Gesundheitsamt die Möglichkeit der beratenden Tätigkeit für das differenzialdiagnostische Vorgehen im Einzelfall besteht und somit in Zukunft Fragen der Kausalität auch prospektiv bearbeitet und damit belastbare Daten zum Zusammenhang von Impfungen und nachfolgender Gesundheitsstörung generiert werden können.

In anderen Ländern bestehen gute Erfahrungen mit der Verknüpfung von Daten aus großen Routinestatistiken, z. B. von Krankenkassen und Krankenhäusern in so genannten „Data Linked Data Bases“ (DLDB) [9, 11]. Auch hier bietet das Infektionsschutzgesetz neue Interventionsmöglichkeiten; erste Modellprojekte zur Erfassung von Impfraten auf der Basis von Krankenkassendaten entstehen aktuell. Hier sollte in $\mathrm{Zu}$ kunft auch die Erfassung von Impfkomplikationen berücksichtigt werden.
Eine zentrale Gutachterkommission, die zu strittigen Fragen der Kausalität Stellung nimmt und einheitliche Bewertungskriterien für die Entschädigungsleistungen erarbeitet und bietet, wird bereits seit Anfang der 1990er-Jahre diskutiert [20]. Diese hat auch heute wie die unterschiedlichen Daten aus den einzelnen Bundesländern zeigen - unvermindert eine Berechtigung und würde einen wertvollen Beitrag zu Transparenz und Vereinheitlichung im Umgang mit Impfkomplikationen leisten können.

\section{Literatur}

1. Chen RT (1999) Vaccine risks: real, perceived and unknown. Vaccine 17:41-46

2. Collet JP, Mac Donald N, Cashman N, Pless R, and the Advisory Committee on Causality Assessment (2000) Monitoring signals for vaccine safety: the assessment of individual adverse event reports by an expert advisory committee. Bull World Health Organization 78:178-186

3. Andrews NJ (2001) Statistical assessment of the association between vaccination and rare adverse events post-licensure.Vaccine 20:49-53

4. Braun M, Ellenberg S (1997) Descriptive epidemiology of adverse events after immunization: reports to the Vaccine Adverse Event Reporting System (VAERS) 1991-1994.J Pediatr 131:529-535

5. Mehta U, Milstien JB, Duclos P, Folb PI (2000) Developing a national system for dealing with adverse events following immunization. Bull World Health Organization 78:170-177

6. Singleton JA, Loyd JC, Mootrey GT, Salive ME, Chen RT, VAERS Working Group (1999) An overview of the vaccine adverse reporting system (VAERS) as a surveillance system. Vaccine 17:2908-2917

7. Annamari P, Paunio M, Kinnunen E, Junttila 0 , Hovi T, Peltola H (2001) Risk of Guillain-Barré syndrom after measles-mumps-rubella vaccination. J Pediatr 138:250-254

8. Chen RT, Glassner JW, Rhodes PH et al. (1997) Vaccine safety datalink project: a new tool for improving vaccine safety Monitoring in the United States. Pediatrics 99:765-773

9. Chen RT, de Stefano F, Davis RL et al. (2000) The vaccine safety datalink: immunization research in the health maintenance organizations in the USA. Bull World Health Organization 78:186-194

10. Zanardi LR, Haber P, Mootrey GT, Niu MT, Wharton M (2001) Intussusception among recipients of rotavirus vaccine: reports to the vaccine advers event reporting system. Pediatrics 107:E 97
11. Niu MT, Erwin DE, Miles Braun M (2001) Data mining in the US Vaccine Adverse event reporting system: early detection of intussusception and other events after rotavirus vaccination. Vaccine 19:4627-4634

12. Murphy TV, Gargiullo PM, Massoudi MS et al. (2001) Intussusception among infants given an oral rotavirus vaccine. $\mathrm{N}$ Engl J Med 344:564-572

13. Verstraeten T, Baughman AL, Cadwell B, Zanardi L, Haber P, Chen RT and the VAERSTeam (2001) Capture-recapture analysis of interssusception after Rotavirus vaccination. Am J Public Health 154:1006-1012

14. Evans $G$ (1999) Vaccine injury compensation programs worldwide. Vaccine 17:25-35

15. Dittmann S (1981) Atypische Verläufe nach Schutzimpfungen. Johann Ambrosius Barth, Leipzig

16. Schneeweiß B, Wiersbitzky S (1991) Schutzimpfungen in der ehemaligen DDR. Sozialpädiatrie 13:422-425

17. Dittmann S, Müller H (1977) Vademekum für Impfärzte. Gustav Fischer, Jena

18. Weibel RE, Benor DE (1996) Reporting vaccineassociated paralytic poliomyelitis: concordance between the CDC and the National vaccine injury compensation Program. Am J Public Health 86:734-737

19. Prevots DR, Sutter RW, Strebel PM, Weibel RE, Cochi SL (1994) Completeness of reporting for paralytic poliomyelitis, United States, 1980 through 1991. Implications for estimating the risk of vaccine-associated disease. Arch Pediatr Adolesc Med 148:479-485

20. Zastrow KD, Schöneberg I, Koch MA (1993) Erfassung und Bewertung von Impfschäden in der Bundesrepublik Deutschland und das Impfschadensregister des BGA. Bundesgesundheitsbl 36:516-518

21. Schöneberg I,Zastrow K-D (1994) Anerkannte Impfschäden in der Bundesrepublik Deutschland 1971-1990. Bundesgesundheitsbl 37:109-112

22. Keller-Stanislawski B, Hartmann K (2001) Existiert ein Zusammenhang zwischen Impfungen und Typ-1-Diabetes mellitus bei Kindern und Jugendlichen. Bundesgesundheitsbl - Gesundheitsforsch Gesundheitsschutz 44:613-618

23. Hans Kuss (2001) Impfconsulting, Mozartstr. 49,65812 Bad Soden, persönliche Mitteilung

24. WHO (2001) Causality assessment of adverse events following immunization. Wkly Epidemiol Rec 12(76):85-92 